\title{
Clinical, histopathological and bacteriological investigations in two cases of relapse following ROM treatment
}

\author{
V. P. SHETTY*, A. V. WAKADE*, N. H. ANTIA*, \\ V. V. PAI $* *$, C. R. REVENKER $* *$, R. GANAPATI** \& \\ H.O. BULCHAND** \\ *The Foundation for Medical Research, 84-A R. G. Thadani Marg, \\ Worli, Bombay 400 018, India \\ **The Bombay Leprosy Project, Vidnyan Bhavan, 11 V. N. Purav \\ Marg, Sion-Chunabhatti, Bombay 400 022, India
}

Accepted for publication 1 September 2000

\section{Introduction}

The efficacy of a single dose of a combination consisting of rifampicin $600 \mathrm{mg}$, ofloxacin 400 $\mathrm{mg}$ and minocycline $100 \mathrm{mg}(\mathrm{ROM})$ for the treatment of single skin lesion paucibacillary (SSL-PB) leprosy has been field tested through multicentre trials in India. ${ }^{1}$ This regimen has been accepted and implemented by the Government of India for single lesion cases of leprosy since July $1997,{ }^{2}$ despite some doubts. ${ }^{3}$ Further use of three, once-monthly dose of ROM was recommended for cases presenting with two to five lesions.

We report here clinical, bacteriological and histopathological findings in two cases, referred with recurrent lesions following release from ROM treatment.

\section{Case 1}

Case 1 (GR), a 40-year-old male, mill worker, was referred to The Foundation for Medical Research (FMR) 2 years after a single dose of ROM treatment. In December 1995, the patient presented with a single coin-sized hypopigmented patch on the right cheek, of 2 months duration. Slit skin smears were negative. He was given a single dose of ROM treatment. The patient was given B complex thereafter. Clinically the patch remained static. In November 1997, there was an increase in the size of the patch, followed by inflammation of the right eye. In January 1998, a punch biopsy was obtained for histopathology and was reported as showing borderline tuberculoid (BT) pathology and the presence of a few acid-fast bacilli (AFB) graded as $1+$ in the section. In May of the same year, the patient was referred to FMR 
for further investigations. On examination, there was a single large hypopigmented, welldefined anaesthetic patch on the right cheek extending up to the lower eyelid. The right eye was inflamed and had diminished corneal sensation. There was no other evidence of any other patches or involvement of major nerves.

Lepromin was given, but slit skin smears were not performed. A deep incision biopsy was obtained from the patch under local anaesthesia. Biopsied tissue was divided into two parts and studied as follows. One part was fixed in Formol Zenker and embedded in paraffin for light microscopy. The second part was homogenized to determine the bacterial load per g. The homogenate thus obtained was injected into the hind footpads of 20 normal S/W mice to determine the viability and sensitivity to rifampicin. Inoculum size used was $0.03 \mathrm{ml}$, containing not more than $1 \times 10^{4}$ Mycobacterium leprae per footpad. Ten mice were given $0.03 \mathrm{~g} \%$ rifampicin through mash feed from day 0 (continuous method ${ }^{4}$ ), and the remaining 10 mice were maintained without any drug as controls. The rifampicin-containing mouse feed was prepared daily in the laboratory. Crushed wheat, milk powder and other essential ingredients were mixed and cooked. The required quantity of drug was mixed into the prepared mash at room temperature using a blender.

In the control group, footpad harvestings were done at months 6,7 and 8. Two per footpad counts were obtained each time. All the remaining mice were harvested at 1 year. The drugtreated mice were taken up for harvesting, on obtaining a significant fold increase in the control mice or at the end of 1 year. ${ }^{4}$

\section{RESULTS}

The lepromin test was negative. Histopathology revealed partial straightening of the rete ridges and the presence of a clear subepidermal zone. Diffused infiltrating cells were seen along the superficial dermis. In the mid- and deep dermis, the infiltrating cells were mostly seen coursing along the adnexa. Several nerve bundles were enlarged and infiltrated both within and around. The infiltrating cells consisted of a mixture of lymphocytes, macrophages that showed mild foamy changes and few epithelioid type macrophages. AFB were seen occasionally in the macrophages and in the nerves. Anti-BCG staining also scored positive $(2+)$. A diagnosis of active BB-BL type of leprosy was made.

The homogenate of the above biopsy also scored positive for AFB. The load was $7 \cdot 55 \times 10^{5} / \mathrm{g}$. initially.

\section{Viability and drug sensitivity}

In control mice harvests carried out at post-inoculation months 6,7 and 8 (two per footpad counts) showed no detectable $M$. leprae. A significant increase $\left(6 \pm 0.3 \times 10^{6}\right.$ fold $)$ in all four footpads (four mice) were obtained at 1 year. The RFP-treated mice, a total of 10 harvests done at 1 year, showed no detectable bacilli, suggesting sensitivity to RFP at the $0.03 \mathrm{~g} \%$ level.

A diagnosis of relapse and probable downgrading was made, probably due to ineffective/ inadequate treatment. His strain of $M$. leprae remained sensitive to rifampicin.

\section{Follow-up}

The patient was retreated in May 1999 with a single dose of ROM. The lesion showed no 
change and was raised and erythematous. Bacteriological index in May 1999 was 2+. Thereafter the patient was put on WHO multidrug therapy for multibacillary leprosy.

\section{Case 2}

Case 2 (SK) was a 50-year-old housewife who was referred to FMR 19 months after ROM treatment with the development of new skin lesions. In June 1996, the patient presented with more than five hypopigmented, anaesthetic patches on the hands and leg. Slit skin smears were negative. She was given three once-monthly supervised doses of ROM treatment. She developed two episodes of type 1 reaction, which were managed with steroids. In April 1998, she reported back with several new lesions and was referred to the FMR. On examination, the new lesions on the face were raised and erythematous. The patches on the back were hypopigmented, both well and ill-defined. The old patches on the hand and leg were mostly dry and ill-defined. All the patches were anaesthetic. There were also large areas of anaesthesia on the dorsum of both the feet. There were no definite palpable tender or thickened nerves. Lepromin test and multiple smears were done. Two of the patches, one old patch from the right forearm and one new patch from the back, were biopsied using local anaesthesia. Parts of the biopsy were processed for histopathology and homogenization for viability and drug sensitivity tests using the standard mouse footpad method as described earlier.

RESULTS

The slit skin smears were negative. The lepromin was moderately positive $(5 \times 4 \mathrm{~mm})$.

\section{Histopathology}

The old patch from the forearm showed a mixture of lymphocytes and oedematous epithelioid cells in the superficial dermis at places touching the epidermal layer. In the mid- and deep dermis, the granuloma was active, more organized and localized around the adnexa. A large number of nerve bundles were also seen that showed infiltration both within and around. The infiltrating cells consisted of a large number of epithelioid type of macrophages and few giant cells surrounded by cuff of lymphocytes. The lesion was diagnosed as active BT leprosy.

In contrast, the biopsy from the new patch on the back revealed a small number of aggregates of poorly defined epithelioid cells surrounded by scanty lymphocytes diagnosed as an indeterminate leprosy. In the Trichrome modified Fite-Faracco (TRIFF) stained sections, intact acid fast bacilli were not seen in either of the lesions. Immunostaining using anti-BCG by the PAP method ${ }^{5}$ showed the presence of distinct intracellular antigen in both the lesions graded as $1+$ and $2+$, respectively.

The homogenate prepared of the biopsy from the forearm (old patch) showed no detectable bacilli $\left(<1 \times 10^{4}\right)$ whereas the patch from back (new patch) showed a bacterial load of $2.9 \times 10^{6} / \mathrm{g}$.

\section{Viability and drug sensitivity}

Both the above homogenates, on passage into the footpads, failed to show any increase at any of the intervals studied in control as well as RFP $(0.03 \mathrm{~g} \%)$ treated mice. However, in view of 
the histopathological findings, which were indistinguishable from any untreated borderline tuberculoid case, this was concluded to be a case of reactivation/relapse, probably due to inadequate treatment.

\section{Follow-up}

From May 1998 to October 1998, the patient was put on steroids with clofazimine. Further information is not available.

\section{Discussion}

A single dose of ROM for SSL-PB has been widely used. Moreover, it has also been extended to cases with two to five lesions. ${ }^{2}$ The two cases presented in this paper are representative examples. The findings in these two cases cast doubts on the efficacy of this regimen. It is evident that in both these cases the treatment received was inadequate. Both cases were diagnosed, treated and monitored by an experienced team of leprologists. In all probability, both would have downgraded subsequently; the clinical as well as the histopathological findings support a downgrading pathology. Patient SK (case 2), who presented with five skin lesions and was smear negative at onset, showed active BT pathology in the old lesion, despite having received three once-monthly doses of ROM, while the new lesion revealed indeterminate pathology and a detectable bacterial count in the homogenate. Lack of $M$. leprae growth in the footpads of normal mice in this case does not rule out relapse or suggest that it is a reactional episode, because not all BT lesions give a positive yield in the footpads of normal mice.

Patient GR (case 1), on the other hand, had presented with a single coin-sized lesion at onset and was smear negative. On relapse, 2 years after release from treatment, the lesion had increased in size and covered the entire cheek. The patient was lepromin negative and the lesion showed a BB-BL pathology. Moreover, the footpad test showed unequivocal growth of M. leprae, which was in keeping with BL pathology. In addition, the inocula was sensitive to $0.03 \mathrm{~g} \%$ rifampicin tested.

These results highlight the importance of obtaining more data in order to judge the true efficacy of ROM treatment even for SSL-PB cases.

\section{Acknowledgement}

Financial support was obtained from Leprosy Mission India and Tata Education Trust Bombay. Ms Jayalaxmi typed the manuscript.

\section{References}

${ }^{1}$ WHO action programme for the elimination of leprosy: efficacy of single dose multi drug therapy for the treatment of single lesion paucibacillary leprosy. Ind J Lepr, 1997; 69: 121-129.

${ }^{2}$ Government of India guidelines on modified MDT. Government of India, Leprosy Division, Directorate General of Health Services, Ministry of Health and Family Welfare, Nirman Bhavan, New Delhi 110 011, India, 1997.

3 Lockwood D. Rifampicin, monocycline and ofloxacin (ROM) for single lesions - what is the evidence. Editorial. Lepr Rev, 1997; 68: 299-300. 
${ }^{4}$ WHO. Laboratory techniques for leprosy. WHO/CDS/LEP, 1987, 86.4.

${ }^{5}$ Sternberger LA, Hardy PH, Cuculis JJ, Mayer HG. The unlabelled antibody enzyme method of immunohistochemistry. Preparation and properties of soluble antigen-antibody complex (horseradish peroxidase antihorseradish peroxidase) and its use in the identification of spirocetes. J Histochem Cytochem, 1970; 18: 315-333. 